\title{
Enviro-economic, facile, one-pot synthesis of novel spiro[pyrazolo $[4,3-c][1,5]$ benzothiazepines] using microwave irradiation
}

\author{
Anshu Dandia, ${ }^{a}{ }^{a}$ Meha Sati, ${ }^{a}$ Kapil Arya, ${ }^{a}$ Pritima Sarawgi, ${ }^{a}$ and André Loupy ${ }^{b}$ \\ ${ }^{a}$ Department of Chemistry, University of Rajasthan, Jaipur 302 004, India \\ ${ }^{b}$ Laboratoire des Réactions Sélectives Sur Supports, ICMMO, UMR 8615, Building 410, \\ Université Paris-Sud, 91405 Orsay cedex, France \\ E-mail: dranshudandia@eth.net; aloupy@icmo.u.psud.fr
}

(received 22 Jun 04; accepted 08 Jan 05; published on the web 18 Jan 05)

\begin{abstract}
An efficient method for the exclusive one-pot synthesis of novel pyrazolo [4,3-c][1,5]benzothiazepines (7a-e) possessing a spiro-3H-indoline nucleus is described. The investigation of the reaction between substituted $\mathrm{o}$-aminothiophenols (5a-e) with 3-pyrazolidinyl-2H-indol-2one (3) to form the title products has been found interesting in view of the fact that different reaction sites are available in the key intermediate 3 which may lead to a mixture of products. Conventionally, 3 did not undergo reactions with 5a-e even under drastic conditions of prolonged reflux using strong acidic or basic catalysts in high boiling organic solvents for many days. However, the exclusive formation of the title products in a satisfactory yield (71\%) was achieved under microwave irradiation coupled with various inorganic supports indicative of a very strong specific microwave effect.
\end{abstract}

Keywords: Pyrazolo [4,3-c][1,5]-benzothiazepines, microwave irradiation

\section{Introduction}

The 1,5-benzothiazepine class of compounds are important as calcium channel blockers of proven utility such as Diltiazem and those in which the fused benzene ring is substituted at various positions have been found to have enhanced pharmacological properties. ${ }^{1}$ A literature survey reveals the enhanced bioactivity of annulated 1,5-benzothiazepines. ${ }^{2-5}$

The research on the chemistry of indoles has been a focus of attention for chemists for a long time, due to their wide spread occurrence in nature and diversified biological activities. ${ }^{6}$ Furthermore, those compounds in which indole-3-carbon is in the form of a spiro atom exhibit enhanced bioactivity. ${ }^{7-9}$ Five and six membered ring containing spiro-3 indole derivatives have been extensively studied ${ }^{10-13}$ but those incorporating a seven membered thiazepine ring have received very little attention. ${ }^{14}$ 
Along with indoles a wide spectrum of pharmacological activities are associated with pyrazole derivatives. ${ }^{15}$ Conventionally, the fusion of a pyrazole ring with a thiazepine nucleus requires harsh conditions such as refluxing with organic solvents in presence of acids or bases. Many research groups have studied the reactions and only three types of pyrazolobenzothiazepines are known, fused at the $\mathrm{b}$ or $\mathrm{c}$ faces of the seven membered ring, requiring multistep tedious synthetic procedures. ${ }^{16-18}$

To the best of our knowledge there is no report on the synthesis of pyrazolo-1,5benzothiazepines incorporating a spiro indole moiety. Hence, for the aforementioned reasons and in a continuation of our search for better and improved cardiovascular drugs, ${ }^{19}$ we investigated the synthesis of novel spiro [indole-pyrazolo[4,3-c][1,5]benzothiazepines] with the assumption that the incorporation of more than one bioactive heterocyclic moiety into a single framework may result into the production of novel heterocycles with enhanced bioactivity.

For this purpose, variously substituted $o$-aminothiophenols $(5$ a-e) were reacted with 3pyrazolidinyl-2H-indol-2-one (3), which is an important synthetic building block for the synthesis of a wide variety of 3 -spiro indolines and condensed indole derivative..$^{20}$ No attention has been paid on the investigation of the above reaction which has been found to be interesting in view of the different reaction sites available in $\mathbf{3}$ which may lead to a number of products depending upon the reaction conditions as studied earlier in reaction of indolylidene chalcones with other nitrogen containing nucleophiles. ${ }^{21}$

Under conventional conditions, no reaction was found to occur between $\mathbf{3}$ and $\mathbf{5 a - e}$ even on extended reflux for many days in organic solvents with strong acidic or basic catalyst (toluene + TFA, ethanol $+\mathrm{HCl}$, piperidine). Conventional synthesis of 1,5-benzothiazepines by the reaction of $o$-aminothiophenols with $\alpha, \beta$-unsaturated carbonyl compounds has been well documented in the past. ${ }^{1,22}$

Microwave-assisted reactions within shorter times are becoming popular for organic chemists $^{23}$ and have recently been reviewed. ${ }^{24}$ More interest has been focused on dry media synthesis under MW irradiation and especially by carrying out the experiments with supported reagents on mineral oxides. ${ }^{25}$ This technology provides a promising alternative to environmentally unacceptable thermal procedures, which are usually time consuming, unsafe and cause solvent emission leading to pollution and waste disposal problems. In many cases, the use of solvent-free methodology or supported reagents under microwaves allows the preparation of products not accessible by the classical heating method.

Hence, in continuation of our earlier interest on the synthesis of various biodynamic spiro-3indole derivatives under MW irradiation ${ }^{26}$ and in an attempt towards the synthesis of novel spiro[indole-pyrazolo [4,3-c] [1,5] benzothiazepines], we studied the reaction of 3 with 5a-e under microwave irradiation coupled with solid supports and under neat conditions. (Scheme 1)

In view of the immense utility of the green synthetic approach, we also carried out the improved neat synthesis of key intermediate (3) under microwave irradiation. A vessel containing neat mixture of isatin (1) and 3-methyl-1-phenyl-2-pyrazolin-5-one (2) was placed on an alumina bath (the temperature of reaction mixture inside the alumina bath reached $102{ }^{\circ} \mathrm{C}$, 
whereas in the neat reaction without using alumina the bath reached only $65^{\circ} \mathrm{C}$ ) and irradiated for 6-8 minutes to give 3 in quantitative yield. TLC studies indicated $100 \%$ conversion of reactants and formation of a single product and were therefore used as such for further reaction. The previously reported ${ }^{27}$ conventional synthesis afforded the major product spiro[dipyrazolopyran-3H-indol]-2H-one (4) along with (3) and required a tedious work-up procedure for isolation of the product. (Scheme 1)

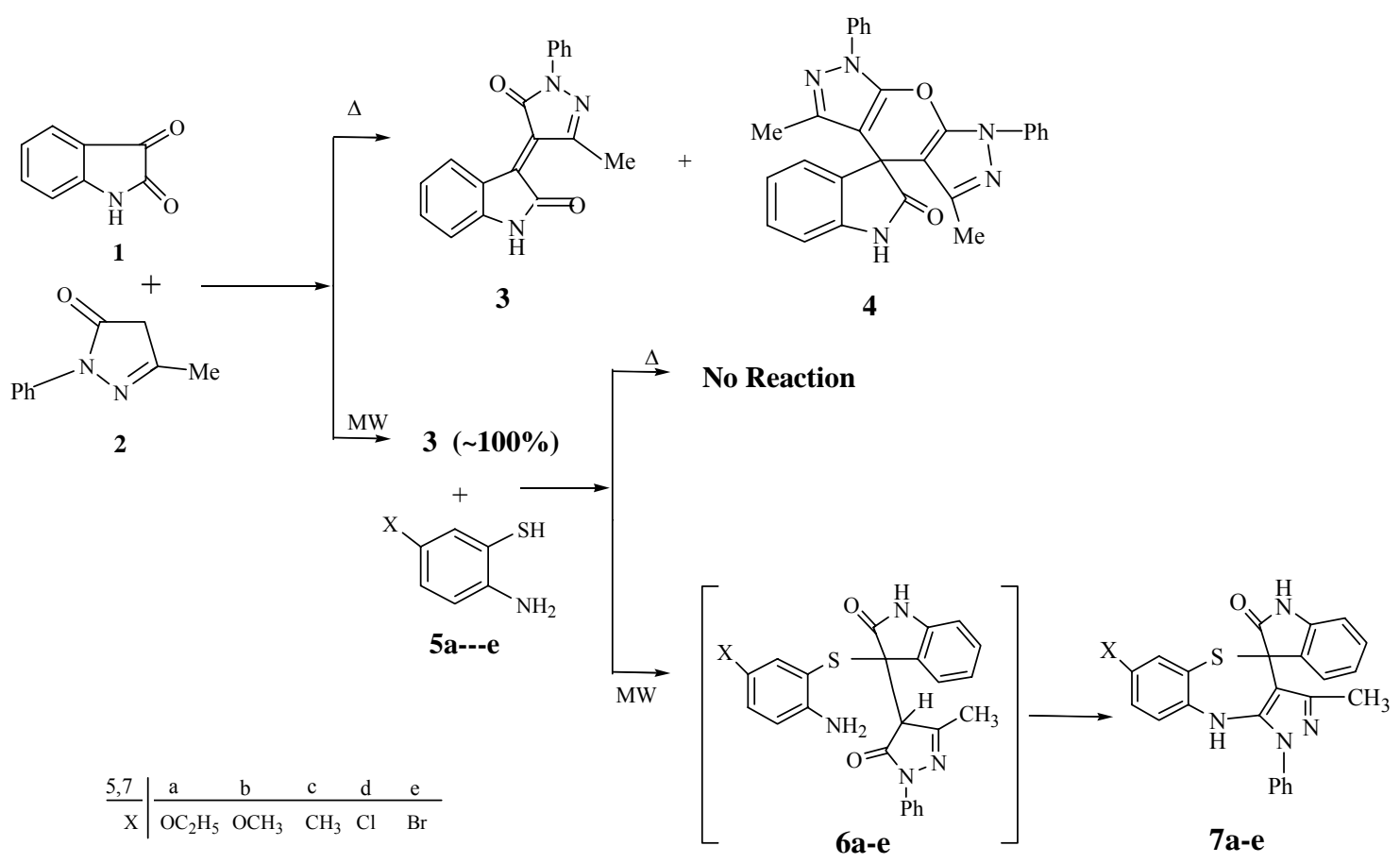

\section{Scheme 1}

Intermediate 3 synthesized 'in situ' was reacted with 5a-e under MW irradiation. In this context suitability of different solid supports was examined that included acidic, basic or neutral alumina, silica, montmorillonite KSF and K10 (Table 1).

The reaction has also been performed under neat conditions (without solvent, support or catalyst), however, no reaction occurred under neat conditions, which could be made successful by adding a few drops of DMF. The role of DMF can be explained as a energy transfer agent and homogenizer to increase the reaction temperature. ${ }^{28}$ This method has an advantage of complete elimination of the use of solvent for absorption of reactant and desorption of product from recyclable solid support. However, the product is formed in comparatively lower yield and purity, which requires further crystallization in this case as compared to solid supported method.

From the results obtained in Table 1, it is clear that montmorillonite K10 is the most adaptable and simplest catalyst for synthesizing 7, since comparatively a higher yield was achieved in shorter reaction time by this method as also observed earlier in clay supported 
reactions .Consequently, this condition was extended to the synthesis of $\mathbf{7 b - e}$. The identity of the compounds synthesized by various methods was confirmed.

Finally, in order to check the possible intervention of 'specific (non-thermal) microwave effects $^{29}$ the best results obtained under microwave irradiation were extrapolated to conventional heating. In the case of compound $7 \mathbf{a}$ the reaction was carried out using a preheated oil-bath, under the same reaction conditions (time, temperature, pressure and vessel). It was found that reaction did not occur and the reactants remained unchanged even on extended reaction times, thus suggesting that the effect of microwaves is not simply thermal. ${ }^{30}$

Table 1. Comparative study for synthesis of $7 \mathbf{a}\left(\mathrm{X}=\mathrm{OC}_{2} \mathrm{H}_{5}\right)$, (A) thermal method (B) MWI method

\begin{tabular}{|c|c|c|c|c|c|c|c|}
\hline Exp. & Thermal Heating & \multicolumn{2}{|l|}{ Time } & \multicolumn{2}{|c|}{ Temp ${ }^{\mathrm{a}}\left({ }^{\circ} \mathrm{C}\right)$} & \multicolumn{2}{|c|}{ Yield $^{\mathrm{b}}(\%)$} \\
\hline 1. & Ethanol + dry $\mathrm{HCl}$ gas & 5 days & & \multicolumn{2}{|l|}{ Reflux } & \multicolumn{2}{|l|}{ Nil } \\
\hline 2. & Toluene + TFA & 5 days & & \multicolumn{2}{|l|}{ Reflux } & \multicolumn{2}{|l|}{ Nil } \\
\hline 3. & Ethanol + piperidine & 5 days & & \multicolumn{2}{|l|}{ Reflux } & \multicolumn{2}{|l|}{ Nil } \\
\hline 4. & Montmorillonite K10 & $7 \mathrm{~min}$ & & \multicolumn{2}{|l|}{142} & \multicolumn{2}{|l|}{ Nil } \\
\hline 5. & Montmorillonite K10 & $420 \mathrm{mir}$ & & \multicolumn{2}{|l|}{142} & \multicolumn{2}{|l|}{ Nil } \\
\hline \multicolumn{8}{|l|}{ B } \\
\hline $\operatorname{Exp}$ & Medium & $\begin{array}{l}\text { MW } \\
\text { (Watts) }\end{array}$ & Power & $\begin{array}{l}\text { Time } \\
\text { (min) }\end{array}$ & Temp $^{\mathrm{a}}$ & $\left.{ }^{\circ} \mathrm{C}\right)$ & Yield $^{\mathrm{b}}(\%)$ \\
\hline 1. & Montmorillonite K10 & 640 & & 7 & 142 & & 71 \\
\hline 2. & Montmorillonite KSF & 640 & & 10 & 138 & & 60 \\
\hline 3. & Acidic Alumina & 640 & & 15 & 135 & & 63 \\
\hline 4. & Basic Alumina & 640 & & 17 & 132 & & 58 \\
\hline 5. & Neutral Alumina & 640 & & 20 & 127 & & 56 \\
\hline 6. & Silica Gel & 640 & & 12 & 142 & & 58 \\
\hline 7. & Neat & 640 & & 15 & 63 & & Nil \\
\hline 8. & Neat $+\varepsilon$ DMF & 640 & & 16 & 112 & & 66 \\
\hline
\end{tabular}

\section{Results and Discussion}

The reaction of $\mathbf{3}$ with $\mathbf{5}$ under microwave irradiation afforded compounds $\mathbf{7}$. The IR spectra of the products 7a-e did not reveal the presence of primary amino group as two bands in the region 3450$3150 \mathrm{~cm}^{-1}$ were absent. On another hand, the presence of absorption bands in the region 3150- 
$3100 \mathrm{~cm}^{-1}(\mathrm{NH})$ and only one carbonyl absorption at $1695-1680 \mathrm{~cm}^{-1}(\mathrm{CONH})$ confirmed the formation of 7a-e instead of the Michael adduct 6.

${ }^{1} \mathrm{H}$ NMR spectra showed a singlet at $\delta 2.68-2.82 \mathrm{ppm}$ assigned to three protons of the methyl group attached to the pyrazole nucleus. Two broad signals at $\delta 8.08-8.12$ and $\delta 8.25-8.30 \mathrm{ppm}$ due to $\mathrm{NH}$ of benzothiazepine and indole respectively were observed which were exchangeable with deuterium. A multiplet due to aromatic protons appeared at $\delta 6.24-7.55 \mathrm{ppm}$. Absence of $\mathrm{NH}_{2}$ and $\mathrm{CH}$ protons also confirmed the formation of spiro products 7.

In the ${ }^{13} \mathrm{C}$ NMR spectrum of the representative compound 7a, sharp signals were observed at $\delta 6.0\left(\mathrm{CH}_{3}\right), 13.9\left(\mathrm{OCH}_{2} \mathrm{CH}_{3}\right) 54.3$ (spiro carbon), $65.8\left(\mathrm{OCH}_{2} \mathrm{CH}_{3}\right), 113-152$ (aromatic ring carbons), $163.4(\mathrm{CONH})$. Formation of the spiro compound 7a was further confirmed by its mass spectrum in which the molecular ion peak $[\mathrm{M}]^{+}$appeared at $\mathrm{m} / \mathrm{z} 454(30.8 \%)$ corresponds to its molecular weight, along with base peak at $411\left[\mathrm{M}^{+}-\mathrm{CONH}\right](100 \%)$.

\section{Experimental Section}

General Procedures. Melting points were determined in open glass capillaries and are uncorrected. Thin layer chromatography on silica gel ' $G$ ' coated glass plates using benzene: ethyl acetate (8:2) as eluent was used for monitoring progress of the reactions. IR spectra $(\mathrm{KBr})$ were recorded on a Magna FT IR-550 spectrophotometer, ${ }^{1} \mathrm{H}$ and ${ }^{13} \mathrm{C}$ NMR spectra $\left[\mathrm{CDCl}_{3}+\right.$ DMSO- $_{6}$ ] were taken on a Jeol FX 90Q spectrometer at 89.55 and $22.49 \mathrm{MHz}$ respectively, using TMS as an internal standard for PMR. Mass spectra were recorded on Jeol D-300 spectrometer at an ionisation potential of 70 e.v. Microwave assisted reactions were carried out on a BPL BMO model, operating at $700 \mathrm{~W}$, generating $2450 \mathrm{MHz}$ frequency 5-substituted-2aminobenzenethiols, ${ }^{31}$ (5a-e) were prepared according to literature reported methods.

1,3-dihydro-3-(2-methyl-5-oxo-4-phenylpyrazolidene)-2H-indol-2-one (3) was synthesized by two routes.

Conventional synthesis. An equimolar mixture of isatin (1) $(0.01 \mathrm{~mol} ; 1.47 \mathrm{gm})$ and 2,4dihydro-5-methyl-2-phenyl-3H-pyrazol-3-one (2) (0.01 mol, $1.74 \mathrm{gm})$ in absolute ethanol $(30 \mathrm{ml})$ was refluxed for 6-8 hours. (TLC indicated formation of two products). The crude product was subjected to crystallization from benzene. A violet coloured compound which was obtained from the benzene soluble portion was identified as 3. M.p., $216^{\circ} \mathrm{C}$ (Rep. ${ }^{27} 218^{\circ} \mathrm{C}$; yield $22 \%$ ). The benzene insoluble portion on recrystallization from ethanol gave white crystals of spiro compound $(58 \%)$. m.p., $172{ }^{\circ} \mathrm{C} .\left(\operatorname{Rep}^{27} 170{ }^{\circ} \mathrm{C}\right)$

Microwave-induced synthesis. A neat mixture of equimolar quantities $(0.001 \mathrm{~mol})$ of $\mathbf{1}$ and $\mathbf{2}$ in a beaker was placed on an alumina bath and irradiated inside a microwave oven for 6 minutes (TLC). The product was extracted with methanol and extract was concentrated on rotoevaporator and left in refrigerator when violet crystals of $\mathbf{3}$ separated, which were found to be 
pure on TLC and used as such for further reactiona. For analytical studies and biological screening it was recrystalized from an appropriate solvent.

Spiro[indole-pyrazolo[4,3-c][1,5]benzothiazepines] (7a-e). Title products 7a-e were prepared by MW-assisted method under solvent-free conditions.

Conventional synthesis. Under thermal conditions, the reaction did not occur in basic (piperidine), acidic (conc. $\mathrm{HCl} / \mathrm{TFA}$ ) and neutral medium (toluene) even on prolonged reflux in high boiling/ volatile organic solvents such as toluene, ethanol for many days.

Microwave assisted synthesis. An equimolar mixture of $3(0.001 \mathrm{~mol}, 0.327 \mathrm{gms})$ and 5a (0.001 mol, 0.169 gms) was adsorbed on solid support [montmorillonite K10/KSF/basic alumina/acidic alumina/ silica gel (20\% by weight of the reactants)] via a solution of methanol. The dry free flowing powder was kept on an alumina bath and irradiated inside the microwave oven for an appropriate time (TLC) (Table-2). The recyclable inorganic solid support was separated after eluting the product with ethanol and excess solvent was evaporated on a rotoevaporator to give crystals of 7a, which were filtered and found pure on TLC.

For analytical and spectral studies the product was recrystallized from ethanol. Likewise the compounds $\mathbf{7 b}$-e were also prepared following the same procedure, using montmorillonite K10 as the solid support.

7a. Yield (91\%), m.p. $=148-150^{\circ} \mathrm{C}$; IR (KBr)/ cm- ${ }^{1}, 3410(\mathrm{NH}), 1690(\mathrm{C}=\mathrm{O}), 1610(\mathrm{C}=\mathrm{N}), 1180$ $(\mathrm{C}-\mathrm{N}) ;{ }^{1} \mathrm{H} \mathrm{NMR}\left(\mathrm{CDCl}_{3}\right) \delta$ ppm $2.69\left(\mathrm{~s}, 3 \mathrm{H}\right.$, pyrazole $\left.\mathrm{CH}_{3}\right), 1.28\left(\mathrm{t}, \mathrm{J}=7 \mathrm{~Hz}, 3 \mathrm{H}, \mathrm{CH}_{3}\right), 4.02(\mathrm{q}$, $\left.\mathrm{J}=7 \mathrm{~Hz}, 2 \mathrm{H}, \mathrm{CH}_{2}\right), 6.25-7.46(\mathrm{~m}, 12 \mathrm{H}, \mathrm{Ar}-\mathrm{H}), 8.08(\mathrm{~s}, 1 \mathrm{H}$, benzothiazepine $\mathrm{NH}), 8.25(\mathrm{~s}, 1 \mathrm{H}$, indole $\mathrm{NH}) \mathrm{D}_{2} \mathrm{O}$ exchangeable; ${ }^{13} \mathrm{CNMR}\left(\mathrm{CDCl}_{3}\right) \delta: 6.0\left(\mathrm{CH}_{3}\right), 13.9\left(\mathrm{OCH}_{2} \mathrm{CH}_{3}\right), 54.3$ (spiro carbon), $65.8\left(\mathrm{OCH}_{2} \mathrm{CH}_{3}\right), 103.6-149.8$ (aromatic carbons), $163.4(\mathrm{CONH}) ; \mathrm{MS}[\mathrm{m} / \mathrm{z}(\%$ rel.int.)]: $454\left(\mathrm{M}^{+}, 30.8 \%\right), 456\left(\left[\mathrm{M}^{+}+2\right], 21.6 \%\right), 452(68.4 \%), 438(74.6 \%), 411\left(\left[\mathrm{M}^{+}-\mathrm{CONH}\right]\right.$ $100 \%$ );Anal. Calc. For $\mathrm{C}_{26} \mathrm{H}_{22} \mathrm{O}_{2} \mathrm{~N}_{4} \mathrm{~S}(\mathrm{MW}=454), \mathrm{C}: 68.69, \mathrm{H}: 4.86, \mathrm{~N}: 12.33$, Found C: 68.70, H: $4.88, \mathrm{~N}: 12.34 \%$.

7b. Yield (88\%), m.p. $=152-154^{\circ} \mathrm{C}$; IR $(\mathrm{KBr}) / \mathrm{cm}^{-}{ }^{1}, 3415(\mathrm{NH}), 1695(\mathrm{C}=\mathrm{O}), 1615(\mathrm{C}=\mathrm{N}), 1168$ $(\mathrm{C}-\mathrm{N}) ;{ }^{1} \mathrm{H} \mathrm{NMR}\left(\mathrm{CDCl}_{3}\right) \delta \mathrm{ppm} 2.73\left(\mathrm{~s}, 3 \mathrm{H}\right.$, pyrazole $\left.\mathrm{CH}_{3}\right), 3.91\left(\mathrm{~s}, 3 \mathrm{H}, \mathrm{OCH}_{3}\right), 6.28-7.52(\mathrm{~m}$, $12 \mathrm{H}, \mathrm{Ar}-\mathrm{H}), 8.10(\mathrm{~s}, 1 \mathrm{H}$, benzothiazepine $\mathrm{NH}), 8.27(\mathrm{~s}, 1 \mathrm{H}$, indole $\mathrm{NH}) \mathrm{D}_{2} \mathrm{O}$ exchangeable; ${ }^{13} \mathrm{CNMR}\left(\mathrm{CDCl}_{3}\right) \delta: 7.8\left(\mathrm{CH}_{3}\right), 55.9$ (spiro carbon), $57.8\left(\mathrm{OCH}_{3}\right), 111.3-149.3$ (aromatic carbons), 151.8(C-OCH$), 164.3(\mathrm{CONH})$; Anal. Calc. For $\mathrm{C}_{25} \mathrm{H}_{20} \mathrm{~N}_{4} \mathrm{O}_{2} \mathrm{~S}(\mathrm{MW}=440), \mathrm{C}: 68.18$, $\mathrm{H}: 4.59$, N: 12.72, Found C: $68.16, \mathrm{H}: 4.58, \mathrm{~N}: 12.69 \%$.

7c. Yield (72\%), m.p. $=260-263^{\circ} \mathrm{C}$; IR $(\mathrm{KBr}) / \mathrm{cm}^{-}{ }^{1}, 3410(\mathrm{NH}), 1680(\mathrm{C}=\mathrm{O}), 1610(\mathrm{C}=\mathrm{N}), 1175$ $(\mathrm{C}-\mathrm{N}) ;{ }^{1} \mathrm{H} \mathrm{NMR}\left(\mathrm{CDCl}_{3}\right) \delta \mathrm{ppm} 2.80\left(\mathrm{~s}, 3 \mathrm{H}\right.$, pyrazole $\left.\mathrm{CH}_{3}\right), 2.30\left(\mathrm{~s}, 3 \mathrm{H}, \mathrm{CH}_{3}\right), 6.44-7.55(\mathrm{~m}, 12$ $\mathrm{H}, \mathrm{Ar}-\mathrm{H}), 8.12(\mathrm{~s}, 1 \mathrm{H}$, benzothiazepine $\mathrm{NH}), 8.25(\mathrm{~s}, 1 \mathrm{H}$, indole $\mathrm{NH}) \mathrm{D}_{2} \mathrm{O}$ exchangeable; ${ }^{13} \mathrm{CNMR}\left(\mathrm{CDCl}_{3}\right) \delta: 7.4\left(\mathrm{CH}_{3}\right), 23.9\left(\mathrm{CH}_{3}\right), 55.9$ (spiro carbon), 109.2-145.4 (aromatic carbons), $164.8(\mathrm{CONH})$; Anal. Calc. For $\mathrm{C}_{25} \mathrm{H}_{20} \mathrm{~N}_{4} \mathrm{O}_{2} \mathrm{~S}(\mathrm{MW}=424), \mathrm{C}: 70.75, \mathrm{H}: 4.73$, N: 13.20, Found C: 70.73, H: 4.75, N: 13.24\%.

7d. Yield (68\%), m.p. $=215-218^{\circ} \mathrm{C}$; IR (KBr) $/ \mathrm{cm}^{-}{ }^{1}, 3400(\mathrm{NH}), 1680(\mathrm{C}=\mathrm{O}), 1620(\mathrm{C}=\mathrm{N}), 1185$ $(\mathrm{C}-\mathrm{N}) ;{ }^{1} \mathrm{H} \mathrm{NMR}\left(\mathrm{CDCl}_{3}\right) \delta \mathrm{ppm} 2.68\left(\mathrm{~s}, 3 \mathrm{H}\right.$, pyrazole $\left.\mathrm{CH}_{3}\right), 6.27-7.39(\mathrm{~m}, 12 \mathrm{H}, \mathrm{Ar}-\mathrm{H}), 8.11(\mathrm{~s}$, $1 \mathrm{H}$, benzothiazepine $\mathrm{NH}), 8.30(\mathrm{~s}, 1 \mathrm{H}$, indole $\mathrm{NH}) \mathrm{D}_{2} \mathrm{O}$ exchangeable; ${ }^{13} \mathrm{CNMR}\left(\mathrm{CDCl}_{3}\right) \delta: 8.2$ 
$\left(\underline{\mathrm{CH}}_{3}\right), 54.8$ (spiro carbon),112.6-148.2 (aromatic carbons),164.7 (ONH); Anal. Calc. For $\mathrm{C}_{24} \mathrm{H}_{17} \mathrm{ON}_{4} \mathrm{SCl}(\mathrm{MW}=444), \mathrm{C}: 64.77, \mathrm{H}: 3.84, \mathrm{~N}: 12.59$, Found $\mathrm{C}: 64.79, \mathrm{H}: 3.85, \mathrm{~N}: 12.61 \%$.

7e. Yield (79\%), m.p. $=138-141^{\circ} \mathrm{C}$; IR (KBr)/ cm- ${ }^{1}, 3405(\mathrm{NH}), 1695(\mathrm{C}=\mathrm{O}), 1615(\mathrm{C}=\mathrm{N}), 1170$ $(\mathrm{C}-\mathrm{N}) ;{ }^{1} \mathrm{H} \mathrm{NMR}\left(\mathrm{CDCl}_{3}\right) \delta \mathrm{ppm} 2.82\left(\mathrm{~s}, 3 \mathrm{H}\right.$, pyrazole $\left.\mathrm{CH}_{3}\right), 6.30-7.60(\mathrm{~m}, 12 \mathrm{H}, \mathrm{Ar}-\mathrm{H}), 8.11(\mathrm{~s}$, $1 \mathrm{H}$, benzothiazepine $\mathrm{NH}), 8.28(\mathrm{~s}, 1 \mathrm{H}$, indole $\mathrm{NH}) \mathrm{D}_{2} \mathrm{O}$ exchangeable; ${ }^{13} \mathrm{CNMR}\left(\mathrm{CDCl}_{3}\right)$ $\delta: 7.5\left(\underline{\mathrm{CH}}_{3}\right), 55.7$ (spiro carbon), 110.4-147.5 (aromatic carbons), 165.3 (ㅁNH); Anal. Calc. For $\mathrm{C}_{24} \mathrm{H}_{17} \mathrm{ON}_{4} \mathrm{SBr}(\mathrm{MW}=488), \mathrm{C}: 58.89, \mathrm{H}: 3.48, \mathrm{~N}: 11.40$, Found $\mathrm{C}: 58.90, \mathrm{H}: 3.50$, $\mathrm{N}: 11.37 \%$.

\section{Acknowledgements}

Financial assistance from CSIR (No 01(1907)/03/EMR-II), New Delhi is acknowledged. We are also thankful to RSIC, CDRI, Lucknow for the elemental and spectral analysis.

\section{References}

1. (a) Levai, A. J. Heterocyclic Chem. 2000, 37, 199. (b) Yamamoto, H.; Asai, H. Chem. Pharm. Bull. 1986, 34, 3844.

2. Chimmiri, A.; Gitto, R.; Grasso, S.; Monforte, A. M.; Zappala, M. Advances in Heterocyclic Chemistry and ref. therein, 1995, 63, 61.

3. De Sarro, G.; Chimiri, A.; Sarro, A. De.; Gitto, R.; Grasso, S.; Zappala, M. Eur. J. Med. Chem. 1995, 30, 925.

4. Steven Walker, D. Eur. Pat. Appl., EP 311, 362, 1989 (C1A61K31/55); U. S. Appl, 1987, 11, $105,244$.

5. Ambrogi, V.; Grandolini, G.; Perioli, L.; Giusti, L.; Lucacchini, A.; Martini, C. Eur. J. Med. Chem. 1995, 30, 429.

6. (a) Abedman, J.; Khalif, A.; Tilt, R.; Martin, S. C.; Suckling, J. J.; Urwin, R.; Waighe, D.; Fishleigh, R. V.; Young, S. C. J. Chem. Res (S) 2000, 264. (b) Cavero, I.; Thirty, C.; Pratz, J.; Lawson, K. Clin. Exp. Hypertens. 1987, A-9, 931; Chem. Abstr. 1987, 107, 191490v. (c) Gruefeld, N. Eur. Pat. Appl. EP 50, 850; Chem. Abstr. 1982, 97, 12749y.

7. (a) Edmonson, S.; Danishefsky, S. J.; Lorenzino, L.; Rosen, N. J. Am. Chem. Soc. 1999, 121, 2147. (b) Mohsen, A. M. G.; Ahmed, El-D.; Nour, M.; Mohammed, A. Bull. Chem. Soc. Jpn. 1999, 72, 471. (c) Maria, I.; Gobor, E.; Maria, K.P.; Csaba, S. Collect. Czech. Chem. Commun. 1999, 64, 408.

8. Joshi, K. C.; Jain, R.; Chand P. Heterocycles 1985, 23, 957.

9. Joshi, K. C.; Joshi, R. J. Indian Chem. Soc. 1999, 76, 515.

10. (a) Wolf, M.; Mascitti, A. A., U.S. Pat. 1969, 3458 525, Chem. Abstr. 1970, 72, 21715p. (b) Giannanigeli, M. J. Heterocyclyl Chem. 1988, 25, 1905. 
11. Rovnyak, G. C.; Narayanan, V. L.; Hauwrtz, R. D., U S. Pat. 4 053 613, 1977; Chem. Abstr. 1978, 88, 22889r.

12. Dandia, A.; Singh, R.; Mérienne, C.; Morgant, G.; Loupy, A. Sulfur Letters 2003, $26,201$.

13. Kirichenko, B. M.; Vladzimirskaya, A. V.; Steblyuk, P. M. Farm. Zh. (Kiev) 1981, 61; Chem. Abstr. 1981, 95, 169060s.

14. (a) Dandia, A.; Upreti , M.; Pant, U. C.; Rani, B. J. Chem. Res. (S) 1998, 752; (M) 1998, 3348. (b) Dandia, A.; Upreti, M.; Pant, U. C.; Rani, B.; Gupta, I. J. J. Fluorine Chem. 1998, $91,171$.

15. Huang, Y. R.; Kalzenellenbogen, J. A. Org. Lett. 2000, 2, 2833.

16. Latrell, R.; Bartmann, W.; Musil, J.; Granzer, E. Ger. Pat. Offen. 2, 707, 269; 1978, Chem. Abstr. 1979, 90, 103949.

17. (a) Maki, Y.; Suzuki, M.; Takaya, M. Chem. Pharm. Bull. 1974, 23, 229. (b) Maki, Y.; Suzuki, M. Jpn. Kokai Tokkyo 1973, 91, 095; Chem. Abstr. 1974, 81, 77984.

18. (a) Ito, I.; Ueda, T.; Oda, N. Chem. Pharm. Bull. 1970, 18, 2058, (b) Ito, I.; Ueda, T. Chem. Pharm. Bull. 1975, 8, 2058.

19. (a) Dandia, A.; Sati, M.; Arya, K.; Sharma, R.; Loupy, A. Chem. Pharm. Bull. 2003, 50, 1137. (b) Dandia, A.; Sati, M.; Arya, K.; Loupy, A. Heterocycles 2003, 60, 563. (c) Dandia, A.; Sati, M.; Loupy, A. Green Chem. 2002, 4, 599. (d) Dandia, A.; Upreti, M.; Saha, M.; Shivpuri, A. Phosphorus, Sulfur and Silicon 1998, 143, 115. (e) Upreti, M.; Pant, S.; Pant, U. C. Indian J. Chem. 1997, 36B, 1181.

20. Dworetzky, S. I. US Pat, 1989, 20020128277; Chem Abstr. 1990, 111, 10356.

21. (a) Joshi, K. C.; Dandia, A.; Sanan, S. Indian. J. Chem. 1991, 30B, 469; ibid. 1990, $29,933$.

(b) Joshi, K. C.; Dandia, A.; Sanan, S. J. Indian. Chem. Soc. 1990, 67, 404. (c) Joshi, K. C.; Dandia, A.; Sanan, S. J. Fluorine Chem. 1989, 44, 59. (d) Joshi, K. C.; Jain, R.; Dandia, A.; Sehgal, V. J. Heterocycl.Chem. 1986, 23, 97.

22. (a) Pant, U.C.; Singhal, B.; Sati, M.; Pant, S. Phosphorus, Sulfur and Silicon 2000, 157, 1.

(b) Somogy, L. Synth. Commun. 1999, 29, 1857. (c) Levai, A. Heterocyclic Commun. 1997, 3, 211 .

23. (a) Katritzky, A. R.; Cai, C.; Suzuki, K.; Singh, S. K. J. Org. Chem. 2004, 69, 811. (b) Katritzky, A. R.; Majumder, S. ARKIVOC 2003, (xiii), 74. (c) Katritzky, A. R.; Singh, S. K. ARKIVOC 2003, (viii), 68. (d) Krutosíková, A.; Lácová, M.; Dandárová, M.; Chovancová, J. Arkivoc 2000, 1, 409. (e) Patel,V. M.; Desai, K. R. ARKIVOC 2004, (i), 123.

24. (a) Hoz, A. D.; Diaz-Ortiz, A.; Moreno, A. Current Organic Chemistry 2004, 8, 903. (b) Nüchter, M.; Ondruschka, B.; Bonrath, W.; Gum, A. Green Chemistry 2004, 6, 128. (c) Loupy, A. In Microwaves in Organic Synthesis, Wiley-VCH: Weinheim, Germany, 2002. (d) Lidstrom, P.; Tierney, J.; Wathey, B.; Westman, J. Tetrahedron 2001, 9225. (e) Varma, R. S. Green Chemistry 1999, 43, 1. (f) Loupy, A.; Perreux, L. Tetrahedron 2001, 57, 9199.

25. (a) Boruah, B.; Boruah, J.; Prajapati, D.; Sandhu, J. C.; Gosh, A. C. Tetrahedron Lett. 1996, 37, 4203. (b) Loupy, A.; Petit, A.; Hamelin, J.; Texier-Boullet, F.; Jacqualt, P.; Mathi, D. 
Synthesis 1998, 1213, (c) Tanaka, K. In Solvent-free organic synthesis Wiley-VCH: Weinheim, Germany, 2003.

26. (a) Dandia, A.; Arya, K.; Sati, M.; Gautum, S. Tetrahedron 2004, 60, 5253. (b) Dandia, A.; Singh, R.; Saha, M.; Shivpuri, A. Die Pharmazie 2002, 57, 602. (c) Dandia, A.; Sachdeva, H.; Singh, R. Synth. Commun. 2001, 31, 1879. (d) Dandia, A.; Sachdeva, H.; Singh, R. J. Chem. Res. (S) 2000, 272. (e) Dandia, A.; Singh, R.; Sachdeva, H.; Arya, K. J. Fluorine Chem. 2000, 111, 61. (f) Dandia, A.; Saha, M.; Rani, B. J. Chem. Res. (S) 1998, 360; (M) 1998, 1425.

27. Joshi, K. C.; Pardasani, R. T.; Dandia, A.; Bhagat, S. Heterocycles 1991, 32, 1491.

28. (a) Marquez, H.; Plutin, A.; Rodriguez, Y.; Perez, E.; Loupy, A. Synth. Commun. 2000, 30, 1067. (b) Suarez, M.; Loupy, A.; Salfran, E.; Moran, L.; Rolando, E. Heterocycles 1999, 51, 21. (c) Perez, R.; Perez, E.; Suarez, ; Gonzalez, L.; Loupy, A.; Jimeno, M. L.; Ochoa, C. Org. Prep. Proced. Int. 1997, 29, 671. (d) Suarez, M.; Loupy, A.; Perez, E.; Moran, L.; Gerona, G.; Morales, A.; Autie, M. Heterocycl. Commun. 1996, 2, 275.

29. (a) Gydye, R. N.; Wei, J. B. Can. J. Chem. 1998, 76, 525. (b) Langa, F.; De la Cruz, P.; De la Hoz, A.; Diaz-Ortiz, A.; Diez-Barra, E. Contemp. Org. Syn. 1997, 4, 373. (c) Preux, L.; Loupy, A. Tetrahedron 2001, 57, 9199. (d) Gabriel, C.; Gabriel, S.; Grant, E. H.; Halsted, B. S.; Mingos, D. M. P. Chem. Soc. Rev. 1998, 7, 213.

30. (a) Syassi, B.; Bougrin, K.; Soufiaoui, M. Tetrahedron Lett. 1997, 38, 8855. (b) Perez, E. R.; Marrero, A. L.; Perez, R.; Autie, M. A. Tetrahedron Lett. 1995, 36, 1779. (c) Varma, R. S.; Varma, M.; Chatterjee, A. K. J. Chem. Soc., Perkin Trans I 1993, 999. (d) Ben Alloum, A.; Labiad, B.; Villemin, D. J. Chem. Soc., Chem. Commun. 1989, 607.

31. Pant, U.C.; Gaur, B. S.; Chugh, M. Indian J. Chem. 1987, 26B, 947. 